\title{
L'école comme centre de la vie communautaire
}

Diversification des représentations et des pratiques dans une communauté semi-rurale d'Afrique du Sud

Ingrid Bamberg

\section{OpenEdition}

1 Journals

Édition électronique

URL : http://journals.openedition.org/etudesafricaines/193

DOI : 10.4000/etudesafricaines. 193

ISSN : $1777-5353$

Éditeur

Éditions de l'EHESS

\section{Édition imprimée}

Date de publication : 1 janvier 2003

Pagination : 121-142

ISBN : 978-2-7132-1809-5

ISSN : 0008-0055

\section{Référence électronique}

Ingrid Bamberg, "L'école comme centre de la vie communautaire », Cahiers d'études africaines [En ligne], 169-170 | 2003, mis en ligne le 20 décembre 2006, consulté le 30 avril 2019. URL : http:// journals.openedition.org/etudesafricaines/193; DOI : 10.4000/etudesafricaines.193 


\title{
Ingrid Bamberg
}

\section{L'école comme centre de la vie communautaire}

\author{
Diversification des représentations et des pratiques \\ dans une communauté semi-rurale d'Afrique du Sud
}

Depuis la mise en place de son nouveau système éducatif unifié ${ }^{1}$, c'est en grande partie sur la décentralisation et en particulier sur le niveau local que mise la politique éducative de l'Afrique du Sud post-apartheid ${ }^{2}$ pour atteindre ses objectifs de réduction des inégalités, d'équité, de participation démocratique et d'accès à un enseignement de qualité pour tous.

En 1996, le South African Schools Act (SASA) institue dans chaque école publique l'un des piliers de la décentralisation de l'éducation, le School Governing Body (SGB). Les SGB sont des comités de gestion composés, d'une part, de membres élus : parents (majoritaires), personnel enseignant et non enseignant et, dans le cas des écoles secondaires, des élèves ; d'autre part, de membres co-optés de la «communauté scolaire». Responsables du recrutement des enseignants et du directeur d'établissement, de la fixation du montant des frais de scolarité, du choix de la langue d'enseignement, des

1. L'Afrique du Sud compte, sous l'apartheid, 19 systèmes éducatifs répartis entre les Blancs, Noirs, Coloureds et Indiens, et les différents homelands et «États indépendants ». Chaque système possède alors son fonctionnement propre, qu'il s'agisse de son mode de financement et d'administration ou des méthodes et des programmes pédagogiques.

2. Nous faisons plusieurs fois référence dans cet article aux catégories « raciales » établies par le régime de l'apartheid pour désigner la population sud-africaine : Noirs, Blancs, Coloured et Indiens. Nous prenons ici le parti de mentionner ces catégories sans guillemets et sans italique. Le lecteur ne doit pas y voir notre adhésion au concept de « race » ni aux critères ayant servis au fondement de ces catégories. Si la limpidité du texte est l'un des facteurs motivant notre choix, nous n'en prenons pas le prétexte pour le justifier. Nous n'accordons aucun bienfondé à ces catégories dans leur acception «raciale». Nous les considérons en tant que facteurs ayant conditionné et conditionnant les dynamiques sociales en Afrique du Sud, d'autant plus que, malgré l'abolition de l'apartheid, elles occupent aujourd'hui encore une place d'importance dans la société sud-africaine, qu'il s'agisse des interactions sociales, des décisions politiques ou de la façon dont les individus eux-mêmes se désignent. 
matières optionnelles, de l'orientation politique et religieuse de l'école, les SGB sont véritablement à la tête de l'école. Véritable « lien entre l'école et la communauté », leur statut ne les cantonne pas cependant à jouer un rôle d'agent de la démocratie. Ils sont, avant tout, partenaires de l'école et ont pour tâche de contribuer à son développement. Autrement dit, le SGB doit fournir une partie des ressources financières de l'école, par le biais des frais de scolarité ou d'opérations de collectes de fonds, et en gérer le budget. En cela, il lui est nécessaire d'inciter la communauté à «participer ».

Après les élections de 1999, la transformation de l'éducation entame, avec le plan d'action intitulé Tirisano, une période qui cible davantage le fonctionnement même de l'établissement scolaire. Parmi les priorités, figure celle de faire des écoles des «centres de la vie communautaire ». Dans un système éducatif décentralisé où le niveau local tient un rôle-clé, faire de l'école un «centre de la vie communautaire » apparaît comme une articulation logique du processus de transformation du système. Toutefois, l'emploi de la notion de communauté nous interpelle.

Tout d'abord, toute école (créée sous l'apartheid) a toujours été attachée à une communauté, ou à un public spécifique, d'une part en termes « raciaux », d'autre part parce qu'une forme d'école communautaire d'initiative locale a existé dans les anciens homelands ${ }^{3}$ (Pampallis 1995), les zones rurales aujourd'hui les plus défavorisées. Ensuite, lorsque Tirisano demande aux SGB de diffuser, en collaboration avec le directeur d'école, la politique et la mission de l'école dans la communauté, tout en précisant que «[les SGB sont] un nouveau concept pour la plupart des différentes communautés du pays ${ }^{4}$, c'est bien aux groupes « raciaux » qu'il est fait allusion.

L'actuelle politique éducative fait donc largement référence à une conception communautariste de la relation entre École et société, selon laquelle il y aurait une communauté définissable dans des limites précises et à laquelle se rattacherait chaque école et où serait exclue toute mobilité sociale et spatiale, et donc toute mixité. La politique éducative reconnaît la multiplicité des communautés qui composent la société sud-africaine mais occulte la diversité des éléments qui peuvent composer une communauté. Cette conception est-elle aujourd'hui acceptable dans le nouveau contexte sud-africain ? À supposer qu'un groupe d'individus liés à une école donnée (parents d'élèves, services sociaux, entreprises...) constitue ce qu'on pourrait appeler la «communauté scolaire», on est amené à se demander en quoi ils forment une communauté et ce qu'ils ont en commun. Si la communauté

3. En raison de l'insuffisance de l'offre scolaire dans les homelands, la population construisait spontanément les écoles. Elle pouvait percevoir une subvention en échange de la construction et de l'entretien des bâtiments scolaires. Contrairement aux actuelles écoles communautaires d'Afrique de l'Ouest, le salaire des enseignants était également couvert par l'État.

4. Les différents systèmes éducatifs « racialement » déterminés sous l'apartheid ont chacun vécu une expérience différente en matière de gestion décentralisée de l'École. 
représente un groupe d'individus défini dans l'espace, comme les habitants d'une localité, en quoi est-elle concernée par une école en particulier ?

Notre propos est ici d'étudier dans quelle mesure l'orientation de la politique éducative très largement axée sur la communauté et sa mise en œuvre au niveau local sont appropriées pour répondre aux objectifs initialement fixés. Nous nous interrogeons sur la notion de «vie communautaire », et plus précisément le concept de communauté, notamment lorsqu'il est appliqué à la politique éducative d'une Afrique du Sud encore fortement imprégnée des conceptions communautaristes de la vie sociale imposées par l'apartheid, mais où les relations entre individus et groupes sont également articulées par de nouvelles dynamiques spatiales et sociales. Nous explorons pour cela les pratiques éducatives possibles des acteurs de l'éducation à travers une étude empirique menée dans une communauté (community) semi-rurale sud-africaine, notamment en mettant en lumière l'importance des représentations de la communauté par les individus. Nous soutenons ici l'hypothèse que l'effet de « routines cognitives » (Derouet 2000) n'est pas sans influence sur les représentations, les décisions et les interactions qui émanent des acteurs de l'École. Nous nous interrogeons donc sur les valeurs et les intérêts communs que défendent les membres de la communauté, sur les modes d'identification au groupe.

Nous explorons ici la notion à travers les deux acceptions suivantes : d'une part la communauté comme ensemble d'individus (parents d'élèves d'une école et habitants d'une même localité) et d'autre part, la communauté en tant qu'espace local. Nous ne faisons pas ce choix parce que nous voyons une cohésion nécessaire entre le cadre spatial et le type de rapports analysés, mais parce que c'est le point de vue qu'adopte la politique éducative sudafricaine dans sa conception de la relation entre École et société. Notre propos est bien, dans la perspective suggérée par A. Henriot-Van Zanten (1990 : 10) de vérifier l'existence et les limites de ce consensus. Il ne s'agit pas de redéfinir le concept de communauté mais de saisir les réalités — ou l'irréalité — de l'école comme «centre de la vie communautaire ».

\section{La relation entre école et communauté dans un contexte décentralisé}

\section{Description de la communauté de l'étude}

Nous avons mené notre étude dans les écoles d'une bourgade rurale qui, depuis le nouveau découpage administratif de l'année 2000, fait partie d'une circonscription (municipality) principalement rurale de près de 15000 ménages, dont $60 \%$ perçoivent un revenu annuel inférieur à R $6001^{5}$. L'agriculture y est le principal secteur d'activité. La bourgade compte 3387 habitants dont 55,7\% sont noirs, $23,4 \%$ coloured, 8,3\% indiens et $12,3 \%$ sont blancs (Demarcation Board 2000).

5. En octobre 2002, un rand est égal à $0,10 €$. 


\section{CARTE I. - LA COMMUNAUTÉ DE L'ÉTUdE : ÉCOLES ET QUARTIERS}

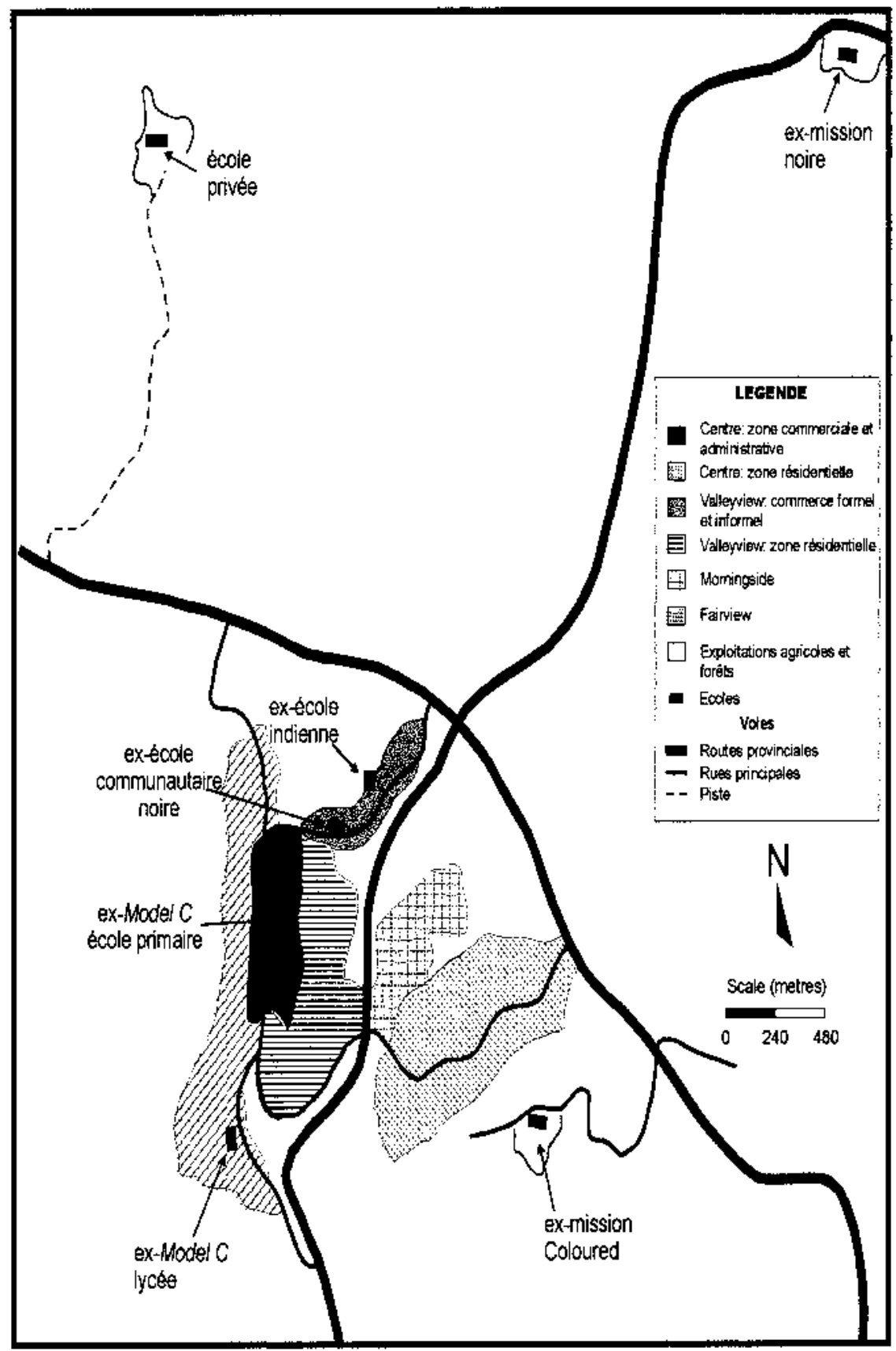

Carte : Ingrid Bamberg, Octobre 2002. 
Anciennement blanche, la localité étudiée présente une répartition des services et de l'habitat dans l'espace encore largement imprégnée de l'organisation territoriale de l'apartheid. La partie haute qui ne comprenait, à sa création, que la bourgade, regroupe une population de classe moyenne, désormais mixte, et où vit la population blanche. Elle rassemble à la fois les services administratifs (mairie, police, poste, télécommunications, antenne locale du département des transports) et les commerces destinés à une population de classe moyenne. La partie basse (Valleyview) a été aménagée dans les années 1980 pour une population principalement coloured et indienne. On y trouve les commerces informels et formels, principalement indiens, qui ciblent une population aux faibles revenus et principalement noire. C'est aussi dans cette zone que se trouve la station de minibus et qu'a lieu la distribution des retraites des personnes âgées en fin de mois ${ }^{6}$. Cette partie de la localité est donc largement investie par la population noire.

En périphérie, deux lotissements composés de logements sociaux (housing scheme) sont séparés du centre par une zone tampon (la route) et peuplés principalement de Coloureds pour l'un (Morningside) et uniquement de Noirs pour l'autre (Fairview). À Morningside, les logements sont des maisons de trois à quatre chambres (avec possibilité d'extension), principalement destinées à des fonctionnaires en raison du revenu mensuel minimum requis (R 3 500) pour bénéficier de la subvention et en raison du montant des remboursements. Les résidents viennent en partie de Valleyview, où ils étaient locataires ; à Fairview, des maisons en dur de deux pièces (two-room houses) forment surtout une base habitable destinée à des familles pauvres mais devant néanmoins percevoir un revenu compris entre R 800 et R 1500 . On $\mathrm{y}$ trouve également un troisième type d'habitat: des maisons en terre (mud houses) de deux pièces bâties par des familles éligibles, et dont la construction à été interrompue au moment du changement de municipalité (local government) en 2000, et qui veulent sauvegarder le lot qui leur avait été attribué. Il y a des familles qui «squattent» en attendant d'obtenir un lot. Les Noirs originaires de diverses zones rurales travaillant dans la bourgade comme domestiques, jardiniers, vendeurs ou ouvriers agricoles dans les fermes avoisinantes ont eu, en principe, un accès prioritaire à ce lotissement. Plus on s'éloigne du centre, plus la population est pauvre. La bourgade et ses lotissements sont entourés d'exploitations agricoles et de forêts. Telle est la géographie de la communauté étudiée (voir carte).

\section{L'offre scolaire}

La localité décrite ci-dessus compte trois écoles primaires, une école offrant les deux cycles et une école secondaire. Chaque école hérite de spécificités

6. Des voitures du département des affaires sociales se déplacent chaque mois pour payer les retraités noirs, qui n'ont généralement pas de compte en banque. Le « jour de la retraite » est un événement mensuel où affluent une foule de retraités, souvent accompagnés, et de vendeurs informels. C'est un jour de grand marché. 
liées à l'administration à laquelle elle était rattachée sous l'apartheid : la population à laquelle l'école était autrefois destinée avec sa "tradition » ou sa «culture » scolaire, sa localisation dans la communauté, l'état des infrastructures et de l'équipement et la composition du personnel enseignant. Elles sont réparties ainsi : l'ancienne école blanche, dite Model C, dans la partie haute du centre, l'ancienne école indienne et l'ancienne école communautaire noire dans la partie basse du centre, l'ancienne mission destinée autrefois aux Coloureds, de l'autre côté de la route, à deux kilomètres environ des nouveaux lotissements. Deux autres écoles, situées à quelques kilomètres, sont rattachées à la communauté : l'ancienne mission destinée autrefois aux Noirs et offrant les deux cycles d'enseignement et une école privée, créée au cours des années 1990, ciblant l'enseignement primaire uniquement.

L'ancienne école Model $C$ et les écoles anciennement administrées par les missions jouissent d'un site scolaire avec terrain de sport, de bureaux pour l'administration quotidienne de l'école (directeur, sous-directeur, responsables pédagogiques des cycles élémentaires et moyens), d'une division du site entre l'espace des cycles élémentaires et moyens, de préaux, de plusieurs classes par niveau. L'ancienne Model $C$ bénéficie d'une piscine et d'une salle informatique. Elle dispose également d'un internat. L'ancienne école indienne et l'ancienne école communautaire noire ont peu d'espace et des infrastructures rudimentaires. La congrégation méthodiste met sa petite église à la disposition de l'école noire pendant la semaine; deux classes se partagent le bas et le haut de l'église. Les locaux lui sont restitués le week-end. Quelques préfabriqués ont été construits ainsi qu'un terrain de sport improvisé dans l'espace limité de la cour de récréation. Le bureau du directeur est toutefois équipé d'un dispositif bureautique de base (ordinateur, imprimante, téléphone, photocopieur), mais obsolète. Malgré les préfabriqués qui constituent les salles de classe, l'ancienne école indienne bénéficie d'un bureau de direction bien équipé et d'un terrain de sport acceptable. L'école privée a été plus récemment construite sur un domaine de 13 ha et bénéficie d'infrastructures et d'équipements sophistiqués : préaux, terrain de sport et équipement sportif, bureaux administratifs, salle informatique, bibliothèque.

La composition «raciale» et le niveau de qualification du personnel enseignant varient également d'une école à l'autre. L'ex-Model C primaire est composée de $36 \%$ d'enseignants coloured, de $28 \%$ d'enseignants noirs et de $36 \%$ d'enseignants blancs. La direction est partagée entre le directeur blanc et le sous-directeur coloured. Les secrétaires sont blanches (2) et coloured (1). Ainsi, le personnel de l'école est à $41 \%$ blanc, $37 \%$ coloured et $22 \%$ noir. La directrice et le sous-directeur de l'ancienne école indienne sont indiens. Le reste du personnel est composé d'enseignants indiens, coloured et noirs. L'ancienne mission coloured compte des enseignants des différents groupes. L'ex-école communautaire noire compte une seule enseignante coloured sur les neuf enseignants de l'école; le reste du personnel 
est noir. L'école privée compte $100 \%$ d'enseignants blancs qui, d'ailleurs, viennent de l'ancienne école Model $C$.

L'offre scolaire dans la communauté de l'étude est homogène du point de vue du statut des écoles; toutes les écoles sont publiques sauf une. En revanche, on peut considérer qu'elle est diversifiée en raison de deux éléments : d'une part, l'appartenance à un ancien département d'éducation selon des critères raciaux lui donne une spécificité en termes d'infrastructures, d'équipements, de niveaux des enseignants, etc. et, d'autre part, la décentralisation au niveau local à travers les SGB accentue cette diversification.

\section{Représentations de la communauté locale}

Les habitants de la communauté auprès desquels ont été conduits les entretiens sont issus des différents quartiers décrits ci-dessus. Dès lors qu'on les interroge sur la communauté en tant que localité, c'est-à-dire en leur demandant d'en décrire l'espace (frontière, quartiers, etc.), une vision globale apparaît nettement: tous les quartiers sans exception sont perçus comme appartenant à la bourgade, en raison de leur interdépendance. L'activité commerciale et administrative située dans le centre (parties haute et basse) est dépendante des nouveaux lotissements qui fournissent la main-d'œuvre et le commerce informel ; l'administration se trouve au centre (ville haute); le pouvoir d'achat se trouve au centre (classe moyenne); certains services sociaux (hôpital) se trouvent à la périphérie ; chaque quartier comprend une zone résidentielle.

La localité est également perçue dans le cadre de la nouvelle démarcation municipale, avec l'ensemble des zones rurales qui la constituent. Elle est alors considérée comme le centre de la circonscription en raison des services qu'elle offre. Selon les habitants interrogés, l'affluence de la population à la recherche de services sociaux ou commerciaux a radicalement changé ses frontières et marque davantage l'appartenance de la bourgade à un ensemble rural qui autrefois était principalement caractérisé par les exploitations agricoles des fermiers blancs. Les habitants interrogés définissent tout naturellement la bourgade comme une "communauté » ou une « localité rurale» (rural community or rural town). L'espace communautaire est donc, selon eux, un espace résidentiel, un lieu d'activités professionnelles, commerciales et d'échanges allant au-delà de sa zone d'habitation propre.

Comme le montrent les témoignages d'une commerçante de Valleyview et d'une résidente de la ville haute, la création des lotissements et la mobilité sociale ont permis davantage de «mélange» dans la communauté.

« Ici, [dans le bourg], il n'y a pas de différences [entre les gens]. Auparavant, nous avions des endroits pour les Blancs et des endroits pour les non-Blancs. Mais maintenant, les gens achètent n'importent où. Vous vous retrouvez avec un voisin 
noir, indien, coloured et que sais-je encore. Donc il n'y a pas beaucoup de différences. »

«Il y a Morningside, Valleyview, Fairview, la section du bas, donc ça fait quatre ; c'est assez grand. Le logement est la plus grande amélioration (dans la communauté), comme toutes ces maisons de deux pièces. Mais il y a encore des gens qui vivent dans des bidonvilles; les autres races se sont éparpillées dans les meilleures parties du bourg. »

Toutefois, les quartiers restent pour la plupart caractérisés par leur étiquette raciale. Les rémanences du cloisonnement social de l'apartheid resurgissent alors dans les descriptions des quartiers. S'y ajoutent les caractéristiques économiques des nouveaux lotissements. La partie de Fairview composée de maisons en terre se voit ainsi affublée des sobriquets de «Chocolate city» ou encore «Eshayamoya» qui, en zulu, signifie « là où les pieds dépassent de la fenêtre ». Les quartiers sont décrits en fonction de leur type d'habitat, du groupe racial qui y réside et de l'activité professionnelle exercée par leurs résidents, illustration de leur situation économique, comme en témoigne ce commentaire d'un habitant de Fairview.

«Le style de vie n'est pas le même. [Le bourg] est un endroit pour les gens riches. Là-bas, il y a des Blancs et ils ont tout. De l'autre côté, il y a Morningside, qui est pour les gens de seconde classe. Vous y trouvez des infirmières, des policiers, des enseignants, etc. Et ici [Fairview], c'est pour les gens pauvres. Donc le style de vie n'est pas le même et il y a beaucoup de délinquance ici. Ils volent des voitures parce qu'ils ont faim. [...] [Le bourg] est plein de commerçants. Morningside c'est pour les infirmières et les gens qui sont allés à l'école. Et ici, vous trouvez des ouvriers en tout genre, des gens qui ne sont pas allés à l'école et qui n'ont pas de certificat. Ici, les gens galèrent. »

Ces caractéristiques sociales et raciales distinguent les quartiers les uns des autres et en excluent, finalement, certains de la communauté globale, comme l'indique cette appréciation d'une commerçante de Valleyview.

«[...] Les maisons juste en dehors du bourg, cet endroit s'appelle Morningside. C'est le nom qu'ils ont donné à ce bourg là-bas. Là où vivent les gens qui ont de faibles revenus, qui gagnent moins de R 2000 . Ensuite il y a les très pauvres, ceux qui n'ont vraiment pas les moyens; ils vivent dans cette partie appelée Fairview. Donc, si quelqu'un me demande si j'habite à Morningside ou à Fairview, je dis simplement que je suis du bourg, sans dire le nom de la rue. [...] Ce sont plutôt les Coloureds et les Noirs qui occupent Morningside. La majorité est coloured. Ces Coloured viennent de villes éloignées [du bourg]. Quand il y a eu le projet de lotissements, ils ont fait des demandes de logement. Ce sont surtout des enseignants qui vivent là-bas. Ce sont des maisons de deux ou trois pièces. Mais les impôts locaux sont moins élevés que ceux que nous payons ici dans le bourg [...].»

On voit bien que le fait d'être originaire d'autres localités rend plus difficile l'intégration des individus et de leur quartier dans la bourgade. Finalement, dès lors que l'on questionne les personnes sur la communauté comme l'ensemble des habitants, on voit que chaque quartier est perçu 
comme une communauté dans la communauté en fonction de caractéristiques raciales et sociales qui tiennent alors lieu de référents objectifs pour marquer la différence et la frontière entre les groupes qui constituent la localité. La réponse d'un habitant de la partie haute à la question de savoir si les habitants de Fairview font partie de la localité en est une illustration:

«Au départ, les lotissements ont été initiés pour les gens des alentours qui n'avaient pas de logement. Mais ils sont venus de toutes parts. Je ne crois pas [qu'ils fassent partie de la localité]. Je pense qu'ils vivent d'une façon tribale. Ils cherchent surtout à améliorer leurs conditions sanitaires et des choses comme ça. Mais c'est leur communauté. Simplement lorsqu'ils viennent faire des courses, ils viennent ici. [...] Ils forment leur propre communauté. »

La communauté est donc éclatée. Il faudrait alors, pour que l'école soit un «centre de la vie communautaire », que chaque établissement soit rattaché à un secteur de la communauté. Si l'on prend en compte leur localisation et leur groupe de rattachement «d'origine», on peut considérer que c'est le cas de l'ancienne Model C (primaire et secondaire), de l'ancienne école indienne, de l'ancienne école communautaire noire et de l'ancienne mission coloured. Or, les pratiques éducatives des individus nous présentent un tout autre schéma quant à la relation entre la localisation de l'école, le groupe de rattachement d'origine et la zone d'habitation des enfants scolarisés.

\section{Stratégies éducatives}

Le tableau I, montre que dans les quartiers du centre et, à la périphérie, dans les zones rurales, une partie de la population ne fréquente plus les écoles de quartier. La population de Fairview effectue également des choix qui incluent l'école la plus proche ainsi que d'autres, plus éloignées. Morningside est un cas différent dans la mesure où ce quartier est le seul à se trouver à égale distance des différentes écoles. Lorsqu'ils sont inscrits en dehors du quartier, on voit également que les enfants ne fréquentent pas une école en particulier, mais sont répartis dans une diversité d'écoles. La communauté du quartier est donc également éclatée en matière de pratiques éducatives et on voit une nette rupture de l'association école-groupe racial du quartier de l'école. Dès lors, en quoi une communauté de quartier estelle concernée par l'école du quartier dans la mesure où elle inscrit ses enfants en dehors du quartier ? En quoi une communauté de quartier, encore largement imprégnée de l'ancien découpage territorial, s'intéresserait-elle à l'école de son quartier dans la mesure où la population inscrite est «étrangère » au quartier ? En quoi les parents qui inscrivent leur enfant dans une école donnée forment-ils une communauté, dans la mesure où ils viennent de quartiers, de groupes « raciaux » et sociaux différents? Le fait d'inscrire 
leur enfant dans la même école suffit-il à en faire une communauté, c'està-dire à ce qu'ils aient quelque chose en commun ?

L'offre est donc exploitée par la demande en fonction de critères autres que la proximité de l'école et qui, même indéterminés, indiquent dans les pratiques des parents en matière de scolarisation des stratégies éducatives qui traduisent l'obsolescence de la vision communautariste du rapport entre École et société. Nous entendons ici le concept de stratégie dans les deux acceptions auxquelles Marie-France Lange et Jean-Yves Martin (1995 : 566) font référence: une finalité qui ordonne des phénomènes étudiés, et cela sans que les acteurs aient nécessairement conscience de l'orientation de leur action; le sens que les acteurs donnent à leur action sur la base d'un choix délibéré. Puisque «parler de stratégies éducatives, c'est se situer au cœur du mouvement social en saisissant les choix fondamentaux d'adaptation et de reproduction des différents acteurs sociaux, depuis les politiques scolaires des Etats jusqu'aux pratiques éducatives des familles » (ibid. : 563-564), nous nous situons, comme ces auteurs, dans une perspective où sont appréhendées tant les stratégies individuelles que collectives.

TABleau I. - OfFre, DEMANDE et PRATiques Éducatives

\begin{tabular}{|c|c|c|c|c|c|}
\hline & $\begin{array}{c}\text { Zone } \\
\text { d'habitation }\end{array}$ & $\begin{array}{l}\text { Population } \\
\text { (majorité) }\end{array}$ & $\begin{array}{c}\text { École } \\
\text { la plus proche }\end{array}$ & $\begin{array}{c}\text { École(s) } \\
\text { fréquentée(s) }\end{array}$ & $\begin{array}{c}\text { Distance } \\
\text { écolel } \\
\text { domicile }\end{array}$ \\
\hline & $\begin{array}{l}\text { Centre, } \\
\text { partie haute }\end{array}$ & Blanche & $\begin{array}{l}\text { - Ex-Model } C \text { primaire } \\
\text { et secondaire }\end{array}$ & - École privée & - $5 \mathrm{~km}$ \\
\hline Centre & $\begin{array}{l}\text { Centre, } \\
\text { partie basse } \\
\text { (Valleyview) }\end{array}$ & $\begin{array}{l}\text { Coloured, } \\
\text { indienne }\end{array}$ & $\begin{array}{l}\text { - Ex-école indienne, } \\
\text { - Ex-école } \\
\text { communautaire noire }\end{array}$ & $\begin{array}{l}\text { - Ex-Model C primaire } \\
\text { et secondaire } \\
\text { - Ex-mission coloured } \\
\text { primaire et secondaire }\end{array}$ & - Quartier \\
\hline \multirow[t]{3}{*}{ Périphérie } & Morningside & $\begin{array}{l}\text { Coloured, } \\
\text { noire }\end{array}$ & $\begin{array}{l}\text { - Ex-mission coloured } \\
\text { primaire et secondaire } \\
\text { - Ex-école indienne } \\
\text { - Ex-école } \\
\text { communautaire noire } \\
\text { - Ex-Model C primaire }\end{array}$ & $\begin{array}{l}\text { - Ex-Model C primaire } \\
\text { et secondaire } \\
\text { - Ex-mission coloured } \\
\text { primaire et secondaire } \\
\text { - Ex-école indienne }\end{array}$ & $\begin{array}{l}\cdot 1-2 \mathrm{~km} \\
\cdot 1-2 \mathrm{~km} \\
\cdot 1-2 \mathrm{~km}\end{array}$ \\
\hline & Fairview & Noire & $\begin{array}{l}\text { Ex-mission coloured } \\
\text { primaire et secondaire }\end{array}$ & $\begin{array}{l}\text { - Ex-mission coloured } \\
\text { primaire et secondaire } \\
\text { - Ex-école } \\
\text { communautaire noire } \\
\text { - Ex-mission noire } \\
\text { primaire et secondaire }\end{array}$ & $\begin{array}{l}\text { - } 1-2 \mathrm{~km} \\
\text { - } 1-2 \mathrm{~km} \\
7,5 \mathrm{~km}\end{array}$ \\
\hline & Zones rurales & Noire & Écoles rurales & $\begin{array}{l}\text { - Ex-Model C primaire } \\
\text { et secondaire, } \\
\text { - Ex-mission coloured } \\
\text { primaire et secondaire } \\
\text { - Ex-mission noire } \\
\text { primaire et secondaire } \\
\text { - Ex-école indienne }\end{array}$ & $\begin{array}{l}20-50 \\
\mathrm{~km}\end{array}$ \\
\hline
\end{tabular}

Source: Enquêtes Ingrid Bamberg, 2002. 
Le tableau II ci-dessous montre ainsi, d'une part, les stratégies éducatives des parents des différents quartiers et, d'autre part, celles des écoles à travers les choix des SGB quant au montant des frais de scolarité et à la langue d'enseignement. Ces deux prérogatives des SGB suffisent à diversifier l'offre scolaire de la communauté et à dissocier les pratiques au sein d'une communauté de quartier. Ainsi, les enfants de Morningside sont inscrits dans presque toutes les écoles; les enfants de Fairview se répartissent dans trois écoles différentes. Sauf dans le cas de l'école privée et de l'ancienne école communautaire noire, la majorité des enfants inscrits dans les écoles sont originaires des zones rurales et non pas des quartiers de la communauté. L'ancienne Model $C$ et l'ancienne école indienne profitent davantage aux enfants des zones rurales qu'à certains enfants de Fairview et Morningside qui sont inscrits à l'ancienne mission noire et qui doivent parcourir $7,5 \mathrm{~km}$ pour se rendre à l'école. C'est en grande partie sur la réputation ou le statut social de l'école que repose la fixation du montant des frais de scolarité et le choix de la langue d'enseignement. Finalement, les écoles pâtissent ou jouissent pour beaucoup $\mathrm{du}$ « statut » qu'elles ont acquis de l'ancien département d'éducation auquel elles étaient rattachées par le passé. Nous montrons ici à travers l'exemple de trois établissements comment l'École segmente « la vie communautaire ».

TABlEAU II. - QUelQues CARACTÉRISTIQUES DE L'OFFRE ET DE LA DEMANDE SCOLAIRES DANS LA COMMUNAUTÉ ÉTUDIÉE

\begin{tabular}{|c|c|c|c|c|c|c|c|}
\hline École & $\begin{array}{l}\text { Situation } \\
\text { spatiale }\end{array}$ & $\begin{array}{c}\text { Frais de } \\
\text { scolarité par } \\
\text { an en Rand }\end{array}$ & $\begin{array}{l}\text { Langue } \\
\text { d'ensei- } \\
\text { gnement }\end{array}$ & $\begin{array}{c}\text { Cycles } \\
\text { d'enseigne- } \\
\text { ment }\end{array}$ & $\begin{array}{l}\text { Origine } \\
\text { des enfants } \\
\text { inscrits }\end{array}$ & $\begin{array}{l}\text { Population } \\
\text { scolaire }\end{array}$ & $\begin{array}{c}\text { Profession } \\
\text { des parents } \\
\text { (en majorité) }\end{array}$ \\
\hline Ex-Model $C$ & $\begin{array}{c}\text { Centre, } \\
\text { partie haute }\end{array}$ & 2640 & Anglais & Primaire & $\begin{array}{c}\text { Centre, } \\
\text { Morningside, } \\
\text { et en majorité } \\
\text { zones rurales }\end{array}$ & $\begin{array}{c}\text { Multiraciale } \\
\text { (majorité } \\
\text { noire) }\end{array}$ & $\begin{array}{c}\text { Fonctionnaires, } \\
\text { entrepreneurs, } \\
\text { commerçants, } \\
\text { fermiers }\end{array}$ \\
\hline $\begin{array}{l}\text { Ex-école } \\
\text { communau- } \\
\text { taire noire }\end{array}$ & $\begin{array}{c}\text { Centre, } \\
\text { partie basse }\end{array}$ & 150 & $\begin{array}{l}\text { Zulu/ } \\
\text { anglais }\end{array}$ & Primaire & Fairview & Noire & $\begin{array}{c}\text { Fonctionnaires, } \\
\text { commerçants } \\
\text { informels, ou- } \\
\text { vriers, domestiques }\end{array}$ \\
\hline $\begin{array}{l}\text { Ex-école } \\
\text { indienne }\end{array}$ & $\begin{array}{c}\text { Centre, } \\
\text { partie basse }\end{array}$ & 550 & Anglais & Primaire & $\begin{array}{c}\text { Zones rurales } \\
\text { en majorité et } \\
\text { Morningside }\end{array}$ & $\begin{array}{l}\text { Multiraciale } \\
\text { (majorité } \\
\text { noire, pas } \\
\text { de Blancs) }\end{array}$ & $\begin{array}{c}\text { Fonctionnaires, } \\
\text { petits } \\
\text { commerçants }\end{array}$ \\
\hline $\begin{array}{l}\text { Ex-mission } \\
\text { coloured }\end{array}$ & $\begin{array}{c}2 \mathrm{~km} \text { des } \\
\text { nouveaux } \\
\text { lotissements }\end{array}$ & 500 & Anglais & $\begin{array}{c}\text { Primaire } \\
\text { et secondaire }\end{array}$ & $\begin{array}{l}\text { Morningside, } \\
\text { Fairview, } \\
\text { centre et } \\
\text { en majorité } \\
\text { zones rurales }\end{array}$ & $\begin{array}{l}\text { Multiraciale } \\
\text { (majorité } \\
\text { noire, pas } \\
\text { de Blancs) }\end{array}$ & $\begin{array}{c}\text { Fonctionnaires, } \\
\text { commerçants } \\
\text { informels, } \\
\text { ouvriers, } \\
\text { domestiques }\end{array}$ \\
\hline $\begin{array}{l}\text { Ex-mission } \\
\text { noire }\end{array}$ & $6,5 \mathrm{~km}$ & $\begin{array}{c}100 \\
\text { (primaire) } \\
350 \\
\text { (secondaire) }\end{array}$ & $\begin{array}{l}\text { Zulu/ } \\
\text { anglais }\end{array}$ & $\begin{array}{c}\text { Primaire } \\
\text { et secondaire }\end{array}$ & $\begin{array}{l}\text { Morningside, } \\
\text { Fairview, } \\
\text { et en majorité } \\
\text { zones rurales }\end{array}$ & Noire & $\begin{array}{c}\text { Fonctionnaires, } \\
\text { commerçants } \\
\text { informels, ou- } \\
\text { vriers, domestiques }\end{array}$ \\
\hline Privée & $5 \mathrm{~km}$ & 10450 & Anglais & Primaire & $\begin{array}{c}\text { Centre } \\
\text { et fermes } \\
\text { avoisinantes }\end{array}$ & $\begin{array}{c}\text { Multiraciale } \\
\text { (majorité } \\
\text { blanche) }\end{array}$ & $\begin{array}{c}\text { Fermiers, } \\
\text { commerçants, } \\
\text { entrepreneurs }\end{array}$ \\
\hline Ex-Model C & $\begin{array}{c}\text { Centre, } \\
\text { partie haute }\end{array}$ & 4950 & Anglais & Secondaire & $\begin{array}{l}\text { Centre, } \\
\text { Morningside, } \\
\text { et en majorité } \\
\text { zones rurales }\end{array}$ & $\begin{array}{c}\text { Multiraciale } \\
\text { (majorité } \\
\text { noire) }\end{array}$ & $\begin{array}{c}\text { Fonctionnaires, } \\
\text { entrepreneurs, } \\
\text { commerçants, } \\
\text { fermiers }\end{array}$ \\
\hline
\end{tabular}

Source : Enquêtes Ingrid Bamberg, 2002. 
L'ancienne école communautaire noire demeure l'école d'une population principalement noire mais, aujourd'hui, principalement pauvre. Lors du décloisonnement racial des écoles, les populations noires de classes moyenne et moyenne supérieure ont retiré leurs enfants des écoles auxquelles elles étaient assignées pour leur offrir un enseignement de meilleure qualité. Un père vivant désormais à Fairview témoigne de ces stratégies d'ascension sociale.

«Les Blancs sont à la tête de l'ancienne mission coloured depuis très longtemps. Il y avait des religieuses allemandes. Elles sont venues ici avec leurs certificats et elles ont enseigné. Ensuite, les enseignants coloured ont pris la relève. Les langues d'enseignement étaient l'anglais et l'afrikaans. Donc, quand les gens ont eu le droit de s'inscrire dans n'importe quelle école, ils ont choisi l'ancienne mission coloured. [...] Les enfants inscrits là-bas peuvent parler anglais très tôt et on peut voir que tel ou tel enfant a été éduqué par un Blanc, un Indien ou un Coloured : des enseignants qui ne parlent pas zulu. »

Les écoles noires ont donc hérité d'une population principalement pauvre dont elles sont souvent «prisonnières » : les SGB sont contraints de fixer des frais de scolarité moindres et ont ainsi de faibles chances d'améliorer l'environnement de l'école et les conditions d'enseignement. Les populations pauvres sont, elles aussi, « condamnées » à ces écoles dont elles peuvent à peine payer les frais de scolarité, ces derniers étant déterminants dans le choix des parents. C'est ainsi que, motivés par des stratégies économiques, une grande partie des habitants de Fairview inscrivent leurs enfants dans l'ancienne école communautaire noire où les frais de scolarité s'élèvent à R 150 par an. Un père de Fairview en témoigne :

«À part l'argent, je ne vois pas d'autre raison de choisir cette école. Les parents aimeraient bien envoyer leurs enfants à [l'ancienne école coloured], mais ils n'ont pas d'argent. Tous les parents veulent la meilleure éducation pour leur enfant. Mais au lieu d'avoir les enfants qui restent avec vous à la maison, c'est mieux de les envoyer dans les écoles bon marché. »

En raison des sureffectifs et du manque de place dans cette école, les parents de Fairview sont aussi contraints de se replier sur l'ancienne mission coloured ou l'ancienne mission noire. Pour certains, c'est tout un calcul pour inscrire, dès le primaire, leur enfant dans l'une de ces écoles, car l'ancienne école communautaire noire se limite au primaire et aucune autre école n'est dans le secondaire équivalente en termes de frais de scolarité. Les enfants qui poursuivent leur scolarité dans le secondaire n'ont alors d'autre choix que l'ancienne mission coloured et l'ancienne mission noire. Pour les autres, l'augmentation sensible des frais de scolarité dans le secondaire est un obstacle pour la poursuite de leurs études.

La langue d'enseignement a une incidence directe sur la mixité - ou la non-mixité — « raciale » de la population scolaire. La langue d'enseignement de l'ancienne école communautaire noire est le zulu avec, officiellement, l'introduction de l'anglais en $3^{\mathrm{e}}$ année et l'enseignement en anglais 
à partir de la $4^{\mathrm{e}}$ année. L'observation du déroulement des cours montre toutefois que la leçon est fixée en anglais au tableau, mais que les enseignants dispensent les leçons en zulu jusqu'à la fin du cycle. Les enfants de Fairview, dont les parents sont vendeurs informels, ouvriers domestiques ou agricoles, agents de nettoyage ou gardiens dans la fonction publique, souvent illettrés et exclusivement zuluphones, forment la majorité de la population scolaire de cette école. Les enseignants voient en partie dans l'illettrisme des parents la raison de leur manque de participation aux activités de l'école et leur désintérêt général pour les affaires scolaires. Cependant, ils font remarquer que les parents assistent plus volontiers aux réunions lorsque l'augmentation des frais de scolarité figure à l'ordre du jour.

L'ancienne Model $C$ peut, quant à elle, se permettre de pratiquer des frais de scolarité équivalents à ceux du passé car la demande est forte. Ancienne école blanche, elle représente la réussite pédagogique et administrative ; une large part du personnel est blanc et donc bien formé. Bien que les enfants blancs de la communauté aient quitté l'ancienne Model $C$ pour l'école privée, la population scolaire multiraciale et en majorité noire ne représente pas un obstacle financier pour l'école. Comme le révèlent les catégories socio-professionnelles des parents qui inscrivent leur enfant dans cette école, les mesures prises par les SGB ciblent une population multiraciale, mais de niveau social équivalent à celui de l'ancienne population scolaire blanche. Elles produisent une sélection parmi la demande scolaire de la communauté et sont déterminantes dans la reproduction sociale. Le commentaire d'un enseignant de l'ancienne Model $C$ en atteste :

«Ils [les élèves de l'école] sont principalement noirs. Nous avons une assez large communauté coloured [ici] et nous attirons un certain nombre d'enfants de ce groupe; ensuite des Indiens et enfin des Blancs. Si l'on considère la population noire, nous attirons principalement des enfants issus de familles de classe moyenne. En fait, nombre d'entre eux viennent en transport de zones extérieures à [la bourgade]. Ils sont aussi un certain nombre à venir du centre ou à être proches du centre. Je parle ici [des zones situées à $20 \mathrm{~km}$ ou $30 \mathrm{~km}$ ]. Ils ont des écoles là-bas, mais... Pour parler clairement, ils ont des écoles "traditionnelles" où ils sont supposés s'inscrire. Mais leurs parents voient notre école comme un centre d'excellence, leurs parents appartenant à la classe moyenne, principalement des enseignants, des infirmières aussi. Certains enfants ont des parents dans le domaine juridique, d'autres dans la médecine. Donc, ce sont des parents progressistes. Et je dois dire que ce sont des parents qui ont un bon niveau d'instruction : ils voient notre école comme un centre d'excellence et ils veulent absolument inscrire leurs enfants à notre école. [...] Si l'on considère le montant de nos frais de scolarité, bien que cela ne soit pas adressé contre les enfants, [...] par le bouche-à-oreille, à travers des discussions, les gens dans la communauté doivent savoir que pour avoir le plaisir de profiter de la qualité de l'enseignement que nous fournissons, cela va leur coûter de l'argent. Et donc, nous n'avons pas beaucoup de parents pauvres qui essaient d'inscrire leur enfant à l'école. »

Cette situation n'est pas sans problèmes, surtout en ce qui concerne la participation des parents à la vie de l'école, comme nous avons pu l'observer 
au cours d'une réunion parents-enseignants. Nous avons ainsi pu comparer une classe multiraciale, dont une partie des enfants vit aux abords de l'école, avec une classe majoritairement noire, dont les enfants viennent de zones rurales. Dans la classe multiraciale, un maximum de $50 \%$ des parents sont venus rencontrer l'enseignant tandis que dans la classe majoritairement noire, seulement $14 \%$ des parents se sont déplacés. Ce manque de participation vaut également lors des activités culturelles ou sportives, comme le montrent ces témoignages d'un membre du personnel et d'une personnalité du centre :

«[...] Dans une petite bourgade comme celle-ci, la plupart des enfants sont inscrits à cette école. Si vous avez un match de football, vous vous attendez à ce que les parents viennent regarder. Si nous avons un match de football ou de hockey, les parents doivent venir regarder. Nous devrions avoir les parents qui regardent, mais ce n'est pas le cas... [...] En fait, la plupart des parents travaillent maintenant, père comme mère. Un bon nombre de nos enfants sont pensionnaires; leurs parents habitent loin et n'ont pas la possibilité de venir voir leur enfant jouer. »

«Ce que l'on voit, c'est que l'implication des parents noirs n'est pas ce qu'était celle des parents blancs ou coloured. Ils ne viennent pas aux réunions et ils n'aident pas lorsqu'il faut récolter des fonds. Lorsqu'il y a un bal de fin d'année, ce n'est pas une fête familiale. Ce genre de journées familiales, de kermesse, toutes ces choses-là sont finies. Je parle de ces activités culturelles et sportives. [...] Je crois que c'est juste une question de ne pas être habitué à s'impliquer. J'ai parlé à un certain nombre d'entre eux, à ceux qui ont participé et ils ont été déçus que les autres ne viennent pas. Ils pensent que le sentiment de ces parents, c'est que tout ça est du ressort de l'école. Tout ce qui concerne le sport, et tout le reste, tout ça ne les regarde pas. À partir du moment où ils ont payé les frais de scolarité, l'enfant va à l'école et il reçoit une bonne éducation. Leur politique est "s'il s'agit de recevoir un prix, je suis là ; si l'enfant gagne quelque chose au sport, je suis fier et je suis là ; mais je ne m'implique dans aucune activité". C'est du genre, “ce n'est pas mon problème, c'est au département d'éducation de s'occuper de tout ça". »

Pour une partie du personnel de l'école comme pour certains parents issus de la communauté blanche, le manque de participation des parents venant des zones rurales vient rompre la relation «traditionnelle » entre l'école et les parents et prive les activités proposées par l'école de leur dimension socialisante. Si les uns l'expliquent par la distance et les difficultés de transports, les autres y voient surtout un manque de collaboration et une apathie qu'ils attribuent à des facteurs «culturels » et à une « perception différente» du mode d'interaction entre l'école et les parents. Les parents seraient donc divisés entre ceux qui s'impliquent en tout point dans les affaires scolaires et ceux pour qui l'école est principalement un terrain de formation, qu'ils payent et qui ne doit pas nécessiter leur concours. Bien que dépeint de manière simpliste, ce clivage entre les parents traduit l'idée non seulement d'une «communauté scolaire» éclatée mais aussi d'une représentation de son éclatement largement influencée par des conceptions communautaristes de la relation école-parents. Il révèle également que l'implication dans les activités offertes par l'école est finalement le fait d'une 
minorité de parents qui n'est pas nécessairement représentative de la population inscrite à l'école et à laquelle incombe la charge du partenariat requis par la politique éducative. Cependant, lorsqu'il s'agit de questions financières et pédagogiques, l'ensemble des parents est prêt à se mobiliser et à s'unir pour une même cause. Le directeur de l'école signale lui aussi que l'assemblée générale, la réunion annuelle au cours de laquelle le bilan financier de l'année, le budget de l'année suivante ainsi que le montant des frais de scolarité sont décidés, remporte des records d'audience. Au cours de l'année, l'école s'est vu imposer par le département d'éducation des «enseignants temporaires obligatoires » (compulsory temporary teachers), c'està-dire des enseignants (noirs) titulaires mais non qualifiés, venant d'écoles noires en zones rurales, et devant se former pendant deux ans auprès des enseignants de l'école en ayant une classe à leur charge. La pétition, lancée sur l'initiative des parents, puis soutenue par le SGB pour renvoyer ces enseignants dans leur école d'origine, n'a pas seulement témoigné de la solidarité de tous les parents de l'école ; elle a également confirmé les stratégies d'ascension sociale des parents noirs, la défense par les parents blancs du niveau scolaire de «leur » école, seul établissement public anciennement blanc des environs et enfin, la mise en commun des efforts de chacun pour satisfaire des stratégies éducatives individuelles.

L'école privée présente un autre cas où la communauté se voit divisée par la question éducative. Ouverte à partir du moment où les autres « races » ont pu accéder à l'ancienne Model $C$, cette école a soulevé et continue à soulever des tensions au sein de la population blanche. Ancienne école blanche dans laquelle les parents blancs ont beaucoup investi financièrement (construction du préau, de la piscine et de la bibliothèque), l'ancienne Model C est passée d'une école considérée comme la propriété de la communauté blanche et gérée par elle à une école publique ouverte à tous et réglementée par un nouveau département d'éducation. La majorité de la population blanche a préféré quitter « son » école et ouvrir une école privée, à $5 \mathrm{~km}$ de la bourgade sur un terrain agricole, inaccessible par transport public et proposant des frais de scolarité de R 950 par mois. Le fait qu'une minorité soit restée «fidèle » à l'ancienne Model C a été à la source de conflits au sein de la communauté blanche. Les uns et les autres sont perçus comme déloyaux, soit vis-à-vis de l'école, soit vis-à-vis de la communauté blanche. Une mère de famille en donne un aperçu :

«J'étais contre [l'école privée] et je pensais que c'était choquant parce que tous les efforts mis dans [cette école] auraient dû être mis dans [l'ancienne Model C], parce que c'est l'école de la communauté.»

La plupart des activités culturelles et sportives étant proposées par les écoles, elles ont été, jusqu'à la scission, le terrain de rencontre de la communauté blanche. L'école a perdu, en ce sens, sa fonction de «centre de la vie communautaire » blanche, comme l'explique une mère : 
« Il y a beaucoup de gens qui vous disent "Mon dieu, comment pouvez-vous mettre votre enfant à l'école publique !" [...] L'école privée divise la communauté. [...] Les enfants ne se rencontrent plus à cause de ça. Donc vous allez à tel endroit tel week-end parce qu'il y a telle compétition. Donc, nous ne sommes plus ensemble. »

Cette stratégie d'évitement, qui reposait initialement sur une idéologie de type communautariste, a donc conduit une partie de la population à se désapproprier l'espace scolaire local, à mettre en exergue des rapports de classes au sein de la population blanche et à créer parmi elle une rupture qui engendre d'autres processus de communalisation. Aux stratégies d'évitement, s'ajoutent des stratégies de distinction que cette population a en commun désormais avec la population indienne. Pour des raisons démographiques, l'école privée a, en effet, connu un déclin de la population blanche et a dû adopter une stratégie économique et accepter des enfants d'autres groupes, dont la majorité est indienne. C'est donc aux côtés des parents indiens que les parents blancs joignent leurs efforts lors de la fête annuelle dont l'objectif premier est de récolter les seuls fonds qui permettent de maintenir les frais de scolarité à leur niveau. La population indienne subit également les conséquences de ces stratégies d'évitement et de distinction. Les activités autour de l'école qui rassemblaient parents et enfants, tendent à les diviser désormais : la scolarisation des enfants indiens à l'école privée distingue les parents qui ont les moyens financiers des autres; les enfants inscrits à l'école privée ne fréquentent plus, l'après-midi, la même madrasa ${ }^{7}$ que les autres.

\section{Décentralisation de l'éducation, vie communautaire et recomposition sociale}

Le manque de participation et d'interaction entre les parents qui sont supposés former une «communauté scolaire » ne peut-il pas alors s'expliquer — au moins en partie — par l'offre scolaire elle-même, par le fait qu'aux yeux des écoles et des SGB, les parents sont exclusivement des « consommateurs d'école », comme en témoigne ce commentaire d'un enseignant de l'ancienne $M o d e l$, se plaignant des parents qui répugnent à payer les frais de scolarité.

«L'école, ce n'est pas gratuit. Si les parents ne sont pas capables d'offrir à leur enfant un enseignement comme celui-ci (de qualité), alors l'école doit être en position de leur recommander une école dont ils peuvent payer les frais de scolarité. Personnellement, j'aimerais bien conduire une Mercedes, mais je n'en ai pas les moyens. Donc, je me contente de quelque chose que je suis en mesure de payer.

7. Les journées scolaires finissant en début d'après-midi, les enfants indiens musulmans suivent un enseignement coranique l'après-midi. 
Faute de grives, on mange des merles, plutôt que d'attendre de l'école et des parents qui payent les frais de scolarité qu'ils prennent en charge ceux qui ne payent pas. »

L'école devient alors un bien marchand et les parents évoluent dans une communauté de marché, qui, si l'on s'en réfère à Weber (1995: 411), est «le plus impersonnel des rapports de la vie pratique dans lesquels les hommes peuvent se trouver », où le marché «n'a de considération que pour les choses, aucune pour les personnes ni pour les devoirs de fraternité ou de piété »; il vient rompre alors l'idéal communautaire et solidaire de la politique éducative. Les parents sont, au contraire, en concurrence. Il apparaît en effet clairement, au cours des entretiens, que l'inscription à l'école procède d'une véritable négociation dans laquelle les parents usent de tactiques relevant du marchandage et du chantage affectif, comme le montrent les témoignages suivants :

«[...] Quand mon enfant a eu fini à [l'ancienne école communautaire noire], ça m'a posé problème parce que je voulais vraiment qu'il continue jusqu'au Bac. J'ai donc demandé au directeur de [l'ancienne mission coloured] de m'aider et d'inscrire mon enfant, même si l'argent n'est pas là. Ils ont refusé. J'ai supplié et finalement ils ont accepté. Ensuite, je leur ai dit que je paierai une certaine somme chaque mois jusqu'à ce que je paie le montant total » (Mère de Fairview).

«Tout ça dépend de la connaissance que vous avez du système. Par exemple, si vous êtes dans cette situation [pauvre], vous pouvez aller voir le directeur de l'école et lui parler... pour discuter avec lui des possibilités de payer les frais de scolarité, peut-être en plusieurs fois si vous n'avez pas la somme exacte. Comme ça l'enfant peut continuer d'aller à l'école, même si les parents n'ont pas payé la somme complète. Le problème, c'est que peu de parents sont au courant de ça et du coup, leurs enfants restent avec eux à la maison parce qu'ils ne payent pas les frais » (Père de Fairview).

De plus, c'est finalement dans le cas où la survie de l'école ou du groupe est en jeu, lorsqu'un intérêt commun, notamment économique, est à défendre (maintien du niveau de l'enseignement par rapport au montant des frais de scolarité engagés, maintien du montant des frais de scolarité, par décision collective ou à travers la collecte de fonds) que l'on voit apparaître les démarches collectives qui peuvent alors être considérées comme des « sociations rationnelles qui s'éteignent avec l'échange des biens qui en font l'objet ». Les parents se retrouvent pour défendre leur bien mais ne s'impliquent pas dans la vie de l'école. On voit que c'est dans le cas de tentatives de re-communalisation (parents blancs de l'école privée), de résistance et de consolidation de l'école comme repère communautaire (parents blancs de l'ancienne Model C) que la participation au rayonnement de l'école existe.

L'idée de faire de l'école un «centre de la vie communautaire » occulte les stratégies éducatives individuelles et inscrit le rapport à l'éducation dans une démarche de type grégaire et uniforme, où la relation entre école et communauté serait déterminée par la proximité et l'appartenance d'un groupe à une école donnée. Ainsi, selon le discours de la politique éducative, 
la relation entre école et communauté serait circonscrite dans un territoire scolaire, où l'espace serait approprié de manière identique à l'espace local. Or, la confrontation des représentations de l'espace local par ses habitants et des stratégies éducatives nous montre, au contraire, des écarts et des distorsions entre l'école choisie et le quartier d'habitation. La communauté locale et la communauté scolaire ne sont pas nécessairement identiques et ne représentent pas nécessairement la même entité; l'interaction entre l'école et des individus vivant à plusieurs dizaines de kilomètres élargit et transforme ladite communauté scolaire. Cette analyse nous révèle un phénomène de désappropriation de l'espace local par certains éléments de la communauté et sa conquête par des éléments extérieurs à la communauté «d'origine » qui ôtent toute réalité à la « vie communautaire ». La communauté n'est pas un ensemble homogène et ne représente pas le collectif que laisse supposer la politique éducative, à travers le SASA et le plan d'action de Tirisano. Ses limites et ses composantes sont un ensemble complexe et ne sont pas aisément identifiables. Ainsi, une école se trouve à la croisée de plusieurs communautés ou groupes communautaires.

Certains éléments semblent former des frontières apparentes de la communauté scolaire : la localisation de l'école dans l'espace, l'association à son ancien département de rattachement et donc à la communauté qu'elle est censée servir «traditionnellement», les caractéristiques physiques des écoles, la composition « raciale »du personnel enseignant et de la direction. Or, ces frontières sont aujourd'hui principalement symboliques dans la mesure où les réalités de l'offre scolaire tels que la langue d'enseignement, les frais de scolarité et l'état des locaux sont à la fois le fruit de décisions prises au niveau local par les SGB et d'un manque d'intervention des autorités éducatives provinciales ou nationales qui accroît les disparités et les inégalités. Les parents noirs et pauvres qui inscrivent leurs enfants dans des écoles délabrées, sous-équipées et aux classes surchargées ne sont motivés ni par un élan communautariste ni par un sentiment d'appartenance. L'autonomie de l'école cantonne les plus pauvres dans un type d'école dont les caractéristiques physiques semblent « correspondre » à leur groupe « communautaire », racial ou social. Or, ne faut-il pas voir dans le choix de ces parents un non choix, une stratégie de survie éducative et, surtout, une telle attente de l'école qu'ils excluent dans leurs stratégies d'éducation le refus de l'institution scolaire ? En effet, comme le rappellent MarieFrance Lange et Jean-Yves Martin (1995: 567) «les stratégies éducatives ne répondent pas seulement à des besoins économiques et n'induisent pas non plus que des comportements sociaux : elles jouent un rôle dans la reproduction sociale, elles s'inscrivent au sein de projets sociétaux ». Quel que soit leur fondement, les stratégies éducatives des parents consultés au cours de l'enquête sont la manifestation à la fois de l'autonomie de l'école, avec son SGB, et de l'absence d'une carte scolaire cohérente et de sectorisation, qui réguleraient le jeu de l'offre et de la demande scolaires. 
Penser l'autonomie de l'école demande alors de questionner le modèle civique sur lequel repose l'institution scolaire (Derouet \& Dutercq 1997) : quel équilibre s'établit entre son statut de « service public localisé » et celui de «petite cité politique locale »? Sans autres mécanismes de redistribution, on voit alors mal comment le «partenariat » préconisé par la politique éducative serait susceptible d'améliorer l'accès à l'éducation et la qualité de l'enseignement et de répondre aux objectifs d'équité et de réduction des inégalités. La politique éducative sud-africaine inscrit l'école dans une logique « civique », dont l'excès de localisme favorise davantage la promotion des intérêts particuliers qu'il n'encourage une démarche citoyenne.

Centre d'études africaines, EHESS, IRD, Paris.

\section{BIBLIOGRAPHIE}

\section{African National Congress}

1994 A Policy Framework for Education and Training.

BAMBERG, I.

2001 «Éducation et démocratisation en Afrique du Sud: le nouveau système éducatif unique et décentralisé face à la reproduction des inégalités », in M.-F. LANGe, « Des écoles pour le Sud », Autrepart, 17 : 91-110.

Buckland, P. \& Hofmeyr, J.

1993 Education Governance in South Africa, Johannesburg, EDUPOL.

Christie, P.

1986 The Right to Learn: The Struggle for Education in South Africa, Johannesburg, SACHED Trust.

Coombe, C. \& Godden, J.

1995 «Local and District Governance in Education : Lessons for South Africa », National Colloquium, Local and District Governance in Education, Johannesburg, Centre for Education Policy.

DEMARCATION BOARD

2000 South African Explorer, CD-Rom.

DEPARTMENT OF EDUCATION

1995 White Paper on Education and Training, Pretoria.

1996 South African Schools Act, Pretoria.

1999 Call to Action, Pretoria.

2000 Tirisano, Implementation Plan 2000-2004, Pretoria. 
Derouet, J.-L.

2000 «La décentralisation, un mouvement national», in L. BouRQuelot, La décentralisation éducative. Visite de chantiers, Paris, INRP («Politiques, pratiques et acteurs de l'éducation ») : 5-11.

Derouet, J.-L. \& DutercQ, Y.

1997 L'établissement scolaire, autonomie locale et service public, Paris, INRP/ ESF.

\section{Education Policy Unit (Natal)}

1993 Report to the National Leadership of the NECC on the Development of a Training Programme for PTSAs, Durban (Unpublished).

Henriot-Van Zanten, A.

1990 L'école et l'espace local. Les enjeux des Zones d'Éducation Prioritaire, Lyon, Presses universitaires de Lyon.

Karlsson, J., Pampallis, J. \& Sithole, S.

1996 Restructuring Education at Sub-national Levels in South Africa, Durban, Education Policy Unit (Natal).

Karlsson, J., Mcpherson, G. \& Pampallis, J.

2001 «A Critical Examination of the Development of School Governance Policy and its Implications for Achieving Equity », in E. Motala \& J. PAmpallis (ed.), The Impact of State Policies on South African Education, Johannesburg, Heinemann : 139-177.

LANGE, M.-F. \& MARTin, J.-Y.

1995 «Les stratégies éducatives en Afrique subsaharienne. Le face à face État/ sociétés », Cahiers des Sciences humaines, 31 (3) : 563-674.

MARCHAND, J.

2000 Les écoles communautaires : Mali, Sénégal, Togo, Paris, Institut international de la planification de l'éducation.

McPherson, G.

2000 «Governance in Public Schools : Four Case Studies», Education Monitor, $11(33): 1-8$.

National Education Policy Investigation

1992 Governance and Administration, Cape Town, Oxford University Press/ NECC.

Nzimande, B. \& Thusi, S.

1998 [1991] «Children of War. The Impact of Political Violence on Schooling in Natal », in Education Policy Unit (Natal), Democratic Governance of Public Schooling in South Africa, a Record of Research and Advocacy from the Education Policy Unit (Natal), Durban, EPU Natal : 3-20. 
PAmpallis, J.

1995 «School Organisation in South Africa: What We Have Inherited and What We Can Do About It », in GreENSteIn et al. (eds), Towards a New Framework for School Organisation, Proceedings of a conference held on 25-26 April 1995, Durban, KwaZulu-Natal, EPU (Natal) and EPU (Wits) : 15-26.

PORTEUS, K.

2001 The Evolution of School Governing Bodies in South African Schooling, Comparative International Education Society Conference, 14-19 March, Washington.

SAyed, Y. \& CARrim, N.

1997 «Inclusiveness and Participation in Discourses of Educational Governance in South Africa », South Africa International Journal of Inclusive Education, 2 (1) : 29-43.

Sithole, S.

1998 [1994] «Parent-Teacher-Student Associations (PTSAs) : Present State and Future Prospects », in Education Policy Unit (Natal), op. cit. : 39-49.

TIKLY, L.

1997 «Redefining Community Involvement in South African Education: The Case for a Greater Role for the "Local Level" in the Finance and Governance of Schools », Oxford International Conference, Education and Development : Education and Geopolitical Change, September 11-15.

UNTERHALTER, E.

1991 «Aspects of Reformism in Bantu Education, 1953-1989 », in E. UNTERHALTER et al., Apartheid Education and Popular Struggles, Johannesburg, Ravan Press : 1-18.

VALLY, S

1998 «Inequality in Education? Revisiting the Provisioning, Funding and Governance of Schooling », Quarterly Review of Education and Training, Wits EPU, 5 (4) : 1-24.

Vally, S. \& Dalamba, Y.

1999 Racism, Racial Integration and Desegregation in South African Public Secondary Schools, Johannesburg, SAHRC.

WEBER, M.

1995 [1971] Économie et société II. L'organisation et les puissances de la société dans leur rapport à l'économie, Paris, Pocket.

WORLD BANK

1992 Governance and Development, Washington D. C., World Bank. 


\section{RÉSUMÉ}

En Afrique du Sud, le système éducatif décentralisé permet désormais aux acteurs locaux de l'éducation de participer aux prises de décision à travers des comités de gestion élus et composés d'enseignants et de membres de la communauté, les parents. Pour les concepteurs de la politique éducative, ces comités constituent le lien indispensable entre l'école et la communauté ainsi qu'un moyen privilégié de faire de l'école un "centre de la vie communautaire » - une priorité gouvernementale depuis la fin des années 1990. Les stratégies éducatives des parents d'une communauté semi-rurale nous montrent comment les décisions de ces comités de gestion en matière de frais de scolarité et de langue d'enseignement modèlent l'offre scolaire et influencent le cadre d'interaction possible entre l'école et la communauté. Elles nous révèlent également les limites de l'idée d'école comme "centre de la vie communautaire » et invitent à nous interroger sur le concept de communauté lorsqu'il est appliqué à la politique éducative d'une Afrique du Sud encore fortement imprégnée des conceptions communautaristes de la vie sociale imposées par l'apartheid, mais où les relations entre individus et groupes sont également articulées par de nouvelles dynamiques spatiales et sociales.

\section{ABSTRACT}

Schools as Centers of Community Life: Ideas and Practices in a Semirural Community in South Africa. - South Africa's decentralized educational system allows locals to take part in decision-making through elected management committees made up of teachers, community members and parents. For those who designed this educational policy, such committees form the indispensable link between the school and community; they turn schools into "centers of community life" a government priority since the late 1990s. Parents, educational strategies in a semi-rural community reveal how the decisions of these management committees about school fees and the language to be used for teaching affect the "supply" of education and influence interactions between the school and community. They also shed light on the limits of schools as centers of community life. Questions arise about applying the concept of a community to educational policy in a nation still deeply imbued with ideas imposed by apartheid about communities and society.

Mots-clés/Keywords: Afrique du Sud, autonomie de l'école, comités de gestion, communauté, décentralisation, éducation, participation, représentations sociales, reproduction sociale, stratégies éducatives/South Africa, education, decentralization, school management committees, school autonomy, community, social reproduction, educational strategies. 